\title{
Synthesis of silicon nanostructures: comparative study
}

\author{
Bassam G. Rasheed \\ Applied sciences department, University of Technology, Baghdad - IRAQ
}

Email address:

dr_bassamghalib@yahoo.com (B. G. Rasheed)

\section{To cite this article:}

Bassam G. Rasheed. Synthesis of Silicon Nanostructures: Comparative Study. Advances in Materials. Vol. 2, No. 1, 2013 , pp. 6-11. doi: 10.11648/j.am.20130201.12

\begin{abstract}
Silicon nanoparticles/nanostructures have been prepared by different methods. Lasers of different operational modes have been employed to prepare silicon nanoparticles by laser-induced etching, laser ablation and laser annealing. Moreover, electrochemical and photoelectrochemical etching were performed to synthesize silicon nanostructures. Optimum etching rate of $2.4 \mu \mathrm{m} / \mathrm{min}$ is obtained for the porous layer prepared by electrochemical etching under optimum conditions of $15 \mathrm{~mA} / \mathrm{cm}^{2}$ and 10 minutes etching current density and etching time, respectively. Characterization of the prepared silicon nanostructures / nanoparticles was carried out using various methods. The experimentally observed Raman spectra of nanostructured layers prepared by three etching techniques reveal a red shift to $518 \mathrm{~cm}^{-1}$ and line broadening of $12 \mathrm{~cm}^{-1}$. While fitting of these spectra with the quantum confinement model provide an average size for nanostructured layers $6,5.5 \mathrm{and} 2 \mathrm{~nm}$ for photochemical, electrochemical and phooelectrochemical etching, respectively. The surface morphology investigation and their analysis provide valuable details on silicon nanostructure/nanoparticle size and size distribution.
\end{abstract}

Keywords: Laser Ablation, Laser Annealing, Silicon Nanostructures

\section{Introduction}

An explosion of interest in silicon nanoparticles was set off in 1990 with Canham's discovery of the photoluminescence (PL) properties from porous silicon which he attributed to the quantum confinement (QC) of carriers [1]. Silicon nanostructures/nanoparticles play a particular role among various materials nanostructures. This may be attributed to many reasons like the low cost, good accessible in addition to low toxicity and fully compatible [2].

Reducing dimensionality in silicon and semiconductors offers fascinating changes in electrical, optical mechanical and electronical properties of the material-based device and the electron mobility becomes very high in these devices. Porous silicon is a complex network of pores separated by thin walls in the nanometer range. The porous structure is classified into three categories; macroporous, mesoporous and nanoporous according to the pores diameters and dimensions [3].

Laser processing of silicon has activated the fabrication of three - dimensional structures in the micron and submicron scale for microelectronics devices. Recently, lasers have also found new applications in microstructuring technology [4]. This technology utilizes lasers to precede individual electronic element of basic patterning processes.

Many works have been carried out on using $\mathrm{CW}$ lasers to synthesize silicon nanostructures [5-8]. Laser-induced etching (LIE) of silicon is a relatively rare technique for creating silicon nanostructures [9] and has been investigated for many applications such as hologram application [10], and grating application [11], which were mainly passive optoelectronic devices. Recently, photochemically etched silicon has been considered for possible active optoelectronic material. Generally, in this process a $\mathrm{Si}$ wafer is immersed in aqueous HF acid and irradiated with laser radiation of appropriate wavelength and power density, electronhole pairs are generated in the irradiated area and a depletion layer is formed. A chemical reaction takes place involving the photo generated holes and the fluorine ions.

Moreover, Nd-YAG laser has a dominant role in precision processing via laser ablation. This technique offers a great possibility of controlling the nanoparticle features since we can easily control the experimental conditions, such as nanoparticle host ambiance and laser parameters [12]. Controlled synthesis of nanoparticles in liquid media could produce a final product in the form of a stable colloid of nanoparticles. The colloidal suspension of silicon nanoparticles consists of different sizes and could exhibits an intense photoluminescence at room temperature. This photoluminescence emission can be explained by the quantum confinement model [1].

The electrochemical etching of silicon is considered as a common technique to produce porous silicon consisting 
silicon nanostructures [13]. The chemical reaction is initiated when the holes supplied by an external power supply at low voltage $[14,15]$. Due to band bending effects at $\mathrm{HF} /$ silicon surface interface, this technique is applicable on p-type conductivity and can be conducted on n-type when the light assisted the process. Therefore, photoelectrochemical etching (light-assisted electrochemical etching) devoted a considerable attention to modify and initiate silicon nanostructures in $\mathrm{p}$ and n-type silicon, respectively [16-18].

Aim of this paper is to highlight the employed techniques to produce silicon nanostructure/nanoparticle and compare between those techniques through the Nano structural features point of view to facilitate selecting silicon nanostructures for various applications.

\section{Experimental Setup}

A commercially available crystalline silicon wafer of p-type and n-type conductivity, orientation $<100>, 2.5$ and $10 \Omega . \mathrm{cm}$ respectively were used to synthesize silicon nanoparticle by different processes. Etching cell made from Teflon has been designed and fabricated as shown in figure (1) to synthesize nanostructured layer at the top of the silicon wafer. This design allows to use the cell for electrochemical, photochemical as well as photoelectrochemical etching. Furthermore, etching only one side of the wafer has been considered in this design, while the backside is completely separated from the etching acid.

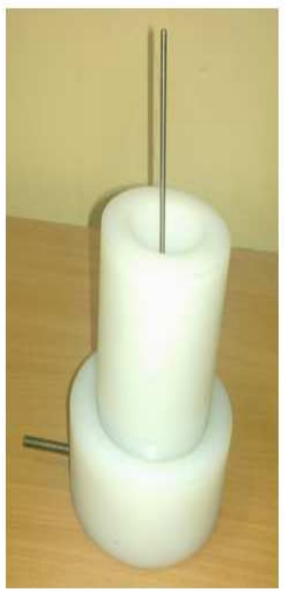

a

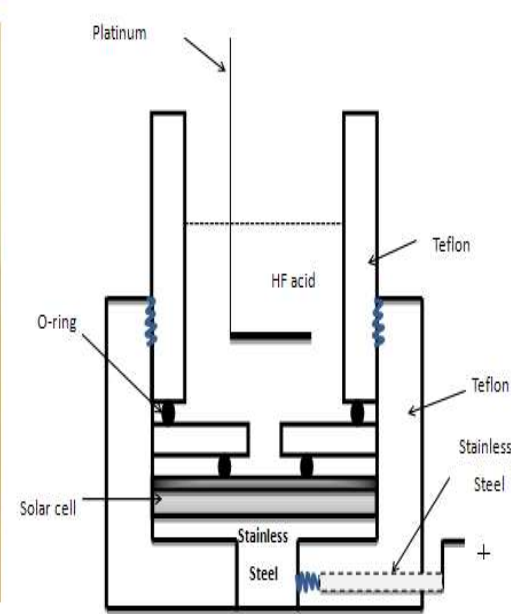

b
Figure 1. The etching cell used in this work a) photograph and b) schematic diagram.

Various lasers of different operational modes were employed in this work. A diode laser of wavelength $(530 \mathrm{~nm})$ and power of $100 \mathrm{~mW}$ was employed for the laser-induced etching process. This laser has high photon energy higher than the silicon band gap energy. Therefore, this laser is appropriate for the laser-induced etching process. While, Q-switched Nd:YAG laser (wavelength of $1.06 \mu \mathrm{m}$ and pulse duration of $10 \mathrm{~ns}$ ) focused to area of $1 \mathrm{~mm}^{2}$ was used for laser ablation. The ablation process was conducted in a vacuum chamber of 0.2 mbar pressure. Moreover, CW $\mathrm{Nd}$ :YAG laser of 1.5 watt was used as a heating source for the laser annealing process.

The scanning electron microscope was used to examine the surface morphology of the prepared layer. While Raman analysis was carried out using Ramanor 210, UiTM, Kuala lumpur, Malaysia.

\section{Results \& Discussions}

Different etching techniques have been employed to produce silicon nanostructures as follows:

\subsection{Eelectrochemical Etching}

Various affecting parameters such as the etching current density, etching time and the HF concentration could affect the nanostructured layer prepared by the electrochemical etching of p-type silicon substrate. To study effects of one parameter, others were kept fixed. The etching current density was varied in the range $\left(5-25 \mathrm{~mA} / \mathrm{cm}^{2}\right)$, while the etching time was ( $5-25$ minutes). The HF acid was also diluted for concentration (40-10\%). It is found that the thickness of the porous layer synthesized by this technique is very small (do not exceed $25 \mu \mathrm{m}$ ) compared with those of photochemical etching. The current density in the electrochemical etching is responsible on holes accumulation at the surface. Farther increase of current densities leads to accumulate holes in much higher speed compared with holes consumption speed and then decreases the porous layer thickness. Figure (2) represents the optical microscope image of the electrochemically prepared porous layer. It is found that the optimum value for the current density correlates to the etching time and the HF concentration. Table I gives the porous layer thickness as well as the etching rate as a function of those parameters. It is found that faster etching rate of $2.4 \mu \mathrm{m} / \mathrm{min}$ is achieved by electrochemical etching under optimum conditions of $15 \mathrm{~mA} / \mathrm{cm}^{2}$, 10 minutes and $10 \%$ HF concentration.
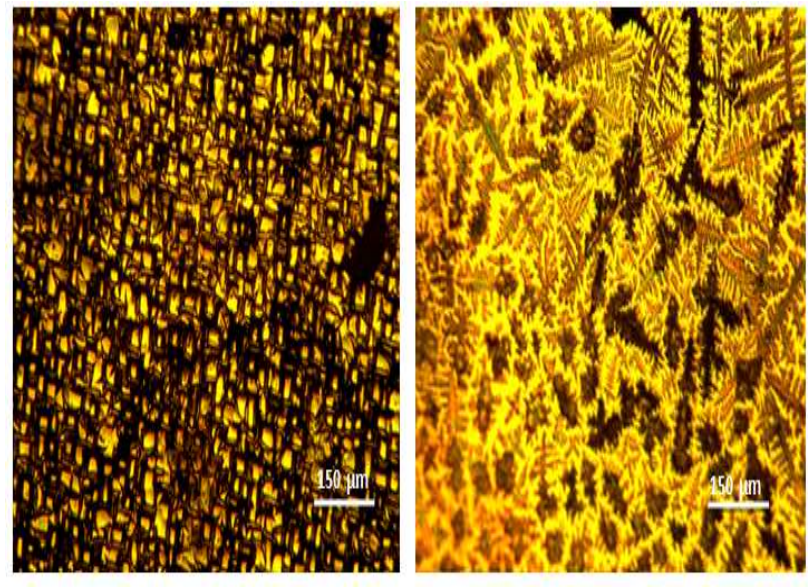

Figure 2. The surface morphology of the electrochemically etched silicon prepared by etching current density of a) 15 and b) $40 \mathrm{~mA} / \mathrm{cm}^{2}$. 
Table 1. The porous layer thickness \& etching rate for different electrochemical etching parameters.

\begin{tabular}{lll}
\hline Parameter & $\begin{array}{l}\text { Porous layer Thickness } \\
(\mu \mathrm{m})\end{array}$ & $\begin{array}{l}\text { Etching } \\
\text { Current density } \\
\left(\mathrm{mA} / \mathrm{cm}^{2}\right)\end{array}$ \\
\hline Etching Current density $\left(\mathrm{mA} / \mathrm{cm}^{2}\right)$ & \\
\hline 5 & 04 & \\
10 & 12 & 0.4 \\
15 & 24 & 1.2 \\
20 & 20 & 2.4 \\
25 & 15 & 2.0 \\
\hline Etching time $(\min )$ & 1.8 \\
\hline 5 & 05 & \\
10 & 24 & 1.0 \\
15 & 20 & 2.4 \\
20 & 12 & 1.2 \\
25 & 08 & 0.7 \\
\hline HF concentration $(\%)$ & 0.3 \\
\hline 10 & 24 & 2.4 \\
20 & 20 & 2.0 \\
30 & 15 & 1.5 \\
40 & 10 & \\
\hline
\end{tabular}
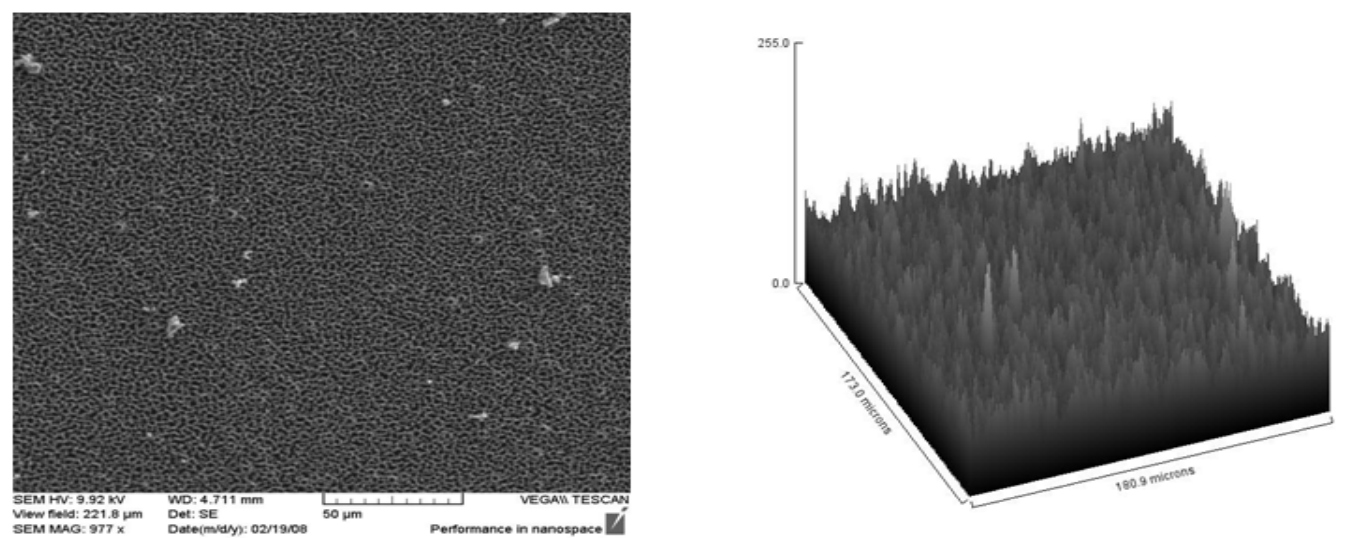

Figure 3. The SEM micrograph for the surface morphology of porous layer prepared by photoelectrochemical etching.
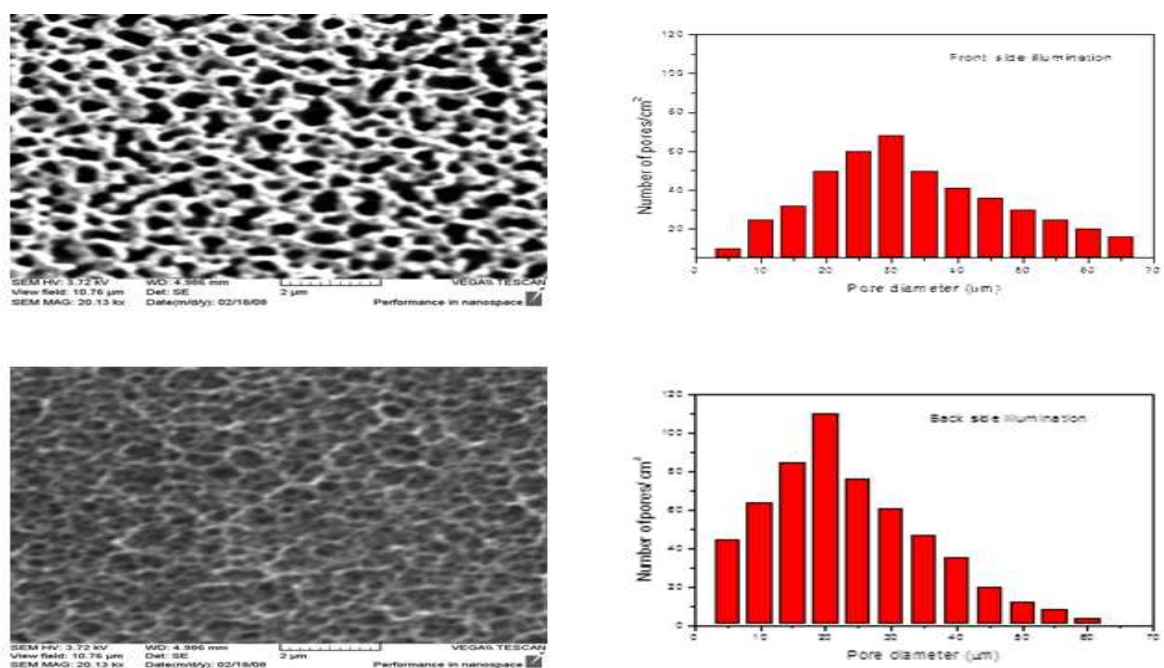

Figure 4. SEM images for the nanostructured porous layer prepared by photoelectrochemical etching with front side illumination (top) and backside illumination (bottom). The right column represents corresponding histograms of their pore distribution. 


\subsection{Photochemical Etching}

In the photochemical etching, the holes which are required in n-type silicon to start the chemical reaction will be generated by light or laser beam. The laser power density is responsible on the photo-generated hole rate [1]. Fast etching rate was observed when high laser power density is employed and holes of fine diameter could be drilled in the wafer at high laser power density (greater than $20 \mathrm{~W} / \mathrm{cm}^{2}$ ) [2]. Therefore, low laser power density is recommended to induce a chemical reaction between silicon substrate and the HF acid and subsequently, form nanostructured layer on the n-type silicon. Moreover, the laser irradiation time is one of the crucial parameter which controls the nanostructured surface features in the photochemical etching technique. It is noticeable that the porous layer thickness produced by this technique is very large (exceed $50 \mu \mathrm{m}$ ) and depends on the preparation parameters.

Furthermore, Lasers could also be employed to produce silicon nanostructures by Laser ablation and Laser annealing. The surface morphology investigation for samples prepared by laser ablation, laser-induced etching and laser annealing were carried out using scanning electron microscope as shown in figure (5). Figure (5-a) reveals formation of micro and nanoparticles synthesized by laser ablation. Laser energy density in the range $5-20 \mathrm{~J} / \mathrm{cm}^{2}$ could be used to ablate silicon micro/nano particles. The surface plot of the collected silicon nanoparticles distributed on a glass substrate is presented in figure (5-a left). It is found that different laser energy could produce different size distribution accordingly; therefore one can adjust and control the required size by using proper laser energy. While, formation of silicon micro and nanocolumns were observed in the porous layer produced by laser-induced etching. Figure ( $5 \mathrm{~b}$ ) shows nanostructured features abundant at the top of micro columns prepared by laser wavelength of $\Lambda=530 \mathrm{~nm}, 10 \mathrm{mi}-$ nutes irradiation time and HF concentration of $10 \%$. The porous layer average thickness is about $70 \mu \mathrm{m}$ which is much higher than those of other techniques. The pore formation in the laser-induced etching process occurs only at the charge carrier (holes) accumulation region and that means the laser-induced etching process is a localized regional technique. Moreover, silicon nanostructures were observed on amorphous silicon film when CW laser beam of suitable power density in the range $\left(100-1000 \mathrm{~W} / \mathrm{cm}^{2}\right)$ is subjected on silicon film to perform laser annealing as shown in figure $(5 \mathrm{c})$. The local heating by laser beam leads to produce spherical silicon nanostructures of different sizes about $5 \mathrm{~nm}-50 \mathrm{~nm}$. These nanostructures have photoluminescence emission in the visible region between $500 \mathrm{~nm}-$ $600 \mathrm{~nm}$ due to the quantum confinement effect [1]. It is found that the photoluminescence peak position depends on the annealing temperature [19].

Raman spectroscopy is one of the most suitable methods for investigating and characterizing silicon nanostructures [20,21]. The Raman scattering effect from silicon nano- structured surfaces could be significantly used to determine the nanostructure features when the Raman scattering model incorporates the phonon quantum confin9ment effect within nanostructures [20]. Therefore, detailed study of Raman scattering line including the line broadening and asymmetry is carried out in this work to determine silicon nanostructures sizes.

Theoretical model involves the phonon quantum confinement model was adopted to fit the experimentally obtained Raman scattering lines. This model is given by the relation [20]:

$$
I(q, L)=\int_{0}^{1} \frac{e^{\frac{-q^{2} L^{2}}{4 a^{2}}}}{\left(\omega-\omega(q)+\left(\frac{\gamma}{2}\right)^{2}\right)} d q
$$

Where $(\boldsymbol{\omega})$ is the phonon wavenumber, $(\gamma)$ is the line width, (a) is the silicon lattice constant and ( $\mathbf{L})$ is the size in nanometer.

Raman lines shape were studied and analyzed using the quantum confinement model for nanostructured surfaces prepared by photochemical (laser-induced etching), electrochemical and photoelectreochemical etching. Table II gives the experimentally obtained Raman line shape analysis involve Raman peak position, line asymmetry and line broadening with theoretically calculated silicon nanocrystallite size by the quantum confinement model. The estimated nanoparticle sizes by fitting the experimental data with the quantum confinement model were $6,5.5$ and $2 \mathrm{~nm}$ (as given in table II) for photochemical, electrochemical and photoelectrochemical etching, respectively. It is found that silicon nanostructures/nanoparticles can be produced by those methods and Raman line shape analysis indicate that the Raman line broadens from $4 \mathrm{~cm}^{-1}$ for the natural crystalline silicon to 8,10 and $12 \mathrm{~cm}^{-1}$ for photochemical, electrochemical and photoelectrochemical etching, respectively. This indicates that wide range of nanoparticles are contribute to Raman scattering for the nanostructured surface produced by photoelectrochemical etching as shown in figure (6).
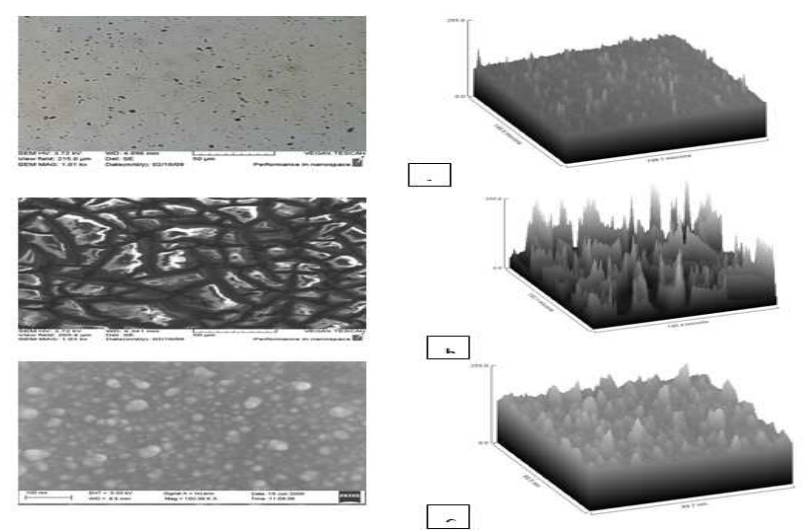

Figure 5. The surface morphology of silicon nanostructures (right column) prepared by laser ablation, laser-induced etching and laser annealing in a, $b$ and $c$ respectively. The left column is the correspondent surface plots. 

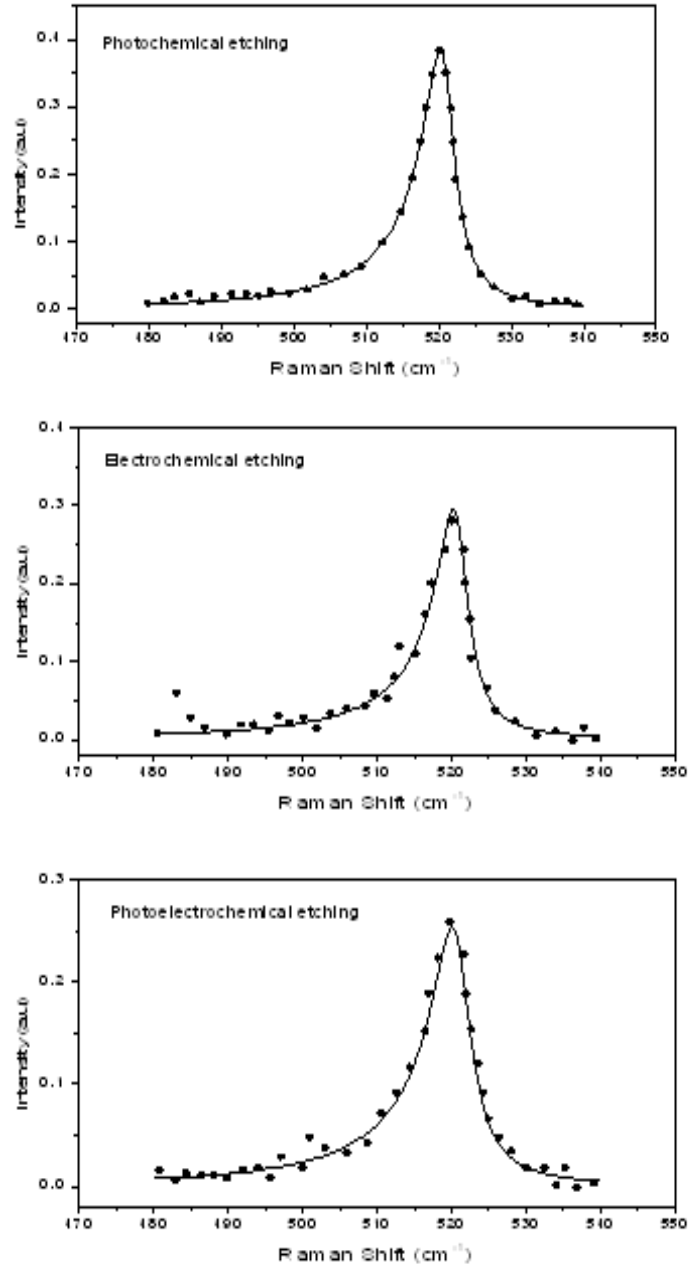

Figure 6. Raman spectra of three etching techniques. The dashed lines represent the experimental data fitted with quantum confinement model (solid lines).

Table 2. The Raman line shape analysis (experimentally obtained) with the estimated nanocrystallite size (theoretically calculated).

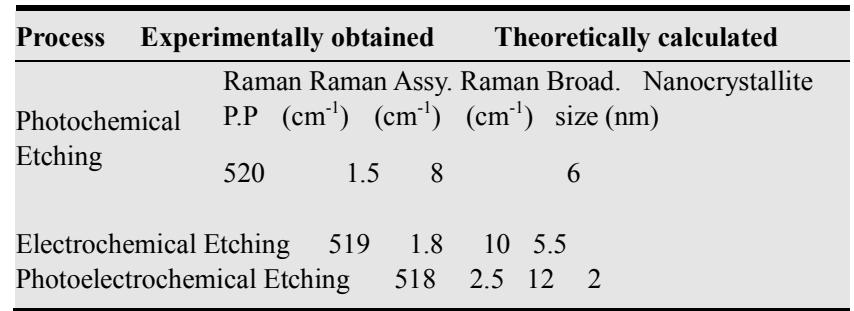

\section{Conclusions}

Lasers could be used to produce silicon nanostructures by different methods. CW visible diode lasers was used to produce silicon nanostructures of controllable morphology by laser-induced etching process, while infrared $\mathrm{CW}$ and pulsed Nd:YAG laser is suitable for synthesis silicon nanostructures by laser ablation and laser annealing, respectively. Laser-induced etching is considered as an alternative technique to produce silicon nanostructures in n-type wafer where the common electrochemical technique is not appli- cable. Thin and thick porous layers could be synthesized by photochemical and electrochemical etching techniques respectively. While backside illumination in photoelectrochemical etching produces homogeneous porous structure of smaller sizes. Raman line shape analysis and the surface morphology could provide valuable information about nanostructured silicon features.

\section{Acknowledgment}

I feel responsible to acknowledge Sandia laboratories/USA and ASTF for their support.

\section{References}

[1] L. Canham,"Silicon quantum wire array fabrication by electrochemical and chemical dissolution of wafers", Appl.Phys.Lett 57, 1990, pp. 1046.

[2] G. John and V. Singh. Effective exponent for the size dependence of luminescence in semiconductor nanocrystallites" Phys. Rev., 263,1995, pp. 3.

[3] A. Loni, L. Canham and P. Calcott. "Blue photoluminescence from rapid thermally oxidized porous silicon following storage in ambient air" J. Appl.Phys., 77, 1995, pp. 3557.

[4] H. Koyama,T. Nakagawa, T. Ozaki and N. Koshida "Post anodization filtered illumination of porous silicon in HF solutions: An effective method to improve luminescence properties" Appl.Phys.Lett.,65,1994, pp. 1656.

[5] L. Koker and K. Kolasiniski, "In situ photoluminescence studies of photochemically grown porous silicon", Mat.Sci. \& Eng B. 69-70, 2000, pp.132.

[6] M. Ngan, K. Lee and K. Cheah, "High power density laser etching of silicon" J.Appl.Phys.83, 1998, pp. 1637.

[7] N. Noguchi and I. Suemune, "Selective formation of luminescent porous silicon by photosynthesis" J.Appl.Phys., 75, 1994, pp. 4765.

[8] K. Cheah, L. Ho and Q. Wang, "Luminescence centers in porous silicon" Appl. Phys.A, 76, 1995, pp. 601.

[9] H. Mavi, B. Rasheed, A. Shukla, S. Abbi, and K. Jain, "Spectroscopic investigations of porous silicon prepared by laser-induced etching of silicon" J.Phys.D:Appl.Phys.34, 2001, 292.

[10] E. Teo, B. Breese, A. Bettiol, D. Karasi, R. Chameaux, F. Watt, and D. Blackwood, "Multicolor Photoluminescence from Porous Silicon Using Focused, High-Energy Helium Ions" Adv. Mater, 18, 2006, pp. 51.

[11] R. Prabakaran, R. Kesavamoorth, and A. Singh, "Optical and microstructural investigations of porous silicon" Mat. Sci. 28, 2005 , pp. 219.

[12] D. Riabinina, C. Durand, M. Chaker and F. Rosei, "Photoluminescent silicon nanocrystals synthesized by reactive laser ablation" Appl.Phys.Lett,88, 2006, pp. 73105.

[13] M. Beale, J. Benjamine and A. Cullis, "Microstructure and formation mechanism of porous silicon" Appl. Phys. Lett. 46, 1985 , pp. 86. 
[14] F. Koch and P. Koch, "Light from Si-nanoparticle systems- a comprehensive view" J. Non-Crys. Solids, 198, 1996, pp. 840.

[15] P. Deak, Z. Hajnal and D. Fuchs, "Correlation between the luminescence and Raman peaks in quantum-confined systems" Thin Solid Films, 255, 1995, pp. 241.

[16] N. Mathews, P. Sebastian and V. Agrawal, "Photoelectrochemical characterization of porous Si", Int.J.Hydr.Enegy, 28, 2003, pp. 629.

[17] M. Thonissen, M. Berger and H. luth, "Preparation of thin porous silicon layers by stain etching", Thin solid Films 276, 1996, 21.
[18] J. Van and H. Nabben, "Processing and Properties of Compound Semiconductors" J.Electrchem. Soc, 38, 1991, pp. 3401 .

[19] A. Abed and B. Rasheed, "Study the Effect of CO2 Laser Annealing on Silicon Nanostructures", Modern Applied Science, 4, 2010, pp. 56.

[20] I. Campbell and P. Fauchet, "Raman Spectroscopy In Low-Dimensional Semiconductors", Solid State and Materials Sciences 14, 1988, pp.S79-S101.

[21] H. Munder, C. Anderzejak and M. ligeon, "A detailed Raman study of porous silicon", Thin Solid Films, 221, 1992, pp. 27. 